material obtained at the Yerkes and McDonald Observatories. Photographic and photovisual magnitudes were obtained from direct photographs taken at the Cassegrain focus of the 82inch McDonald telescope. To calibrate these photographs, a sequence of stars in the field was observed photoelectrically. A number of stars of the North Polar Sequence were also observed with the photoelectric photometer, partly to relate the observed colors to the international system, partly to establish the zero of the calibration sequence and to reduce the observed photovisual magnitudes to the NPS scale.

During 1948, direct photographs of M I5 were taken with the 40-inch refractor at the Yerkes Observatory. These were compared with plates taken by Ritchey and Barnard between I900 and I9Io in order to obtain proper motions. The proper motions were then used to separate foreground stars from members of the cluster and thus to eliminate extraneous stars from the colormagnitude diagram.

The resulting color-magnitude diagram shows a well-defined "red giant" sequence extending from zero absolute magnitude with color index approximately 0.65 to -2.2 absolute magnitude with color index approximately I.4. There are a few scattered stars on the blue side of this "red giant" sequence. One of these objects is known to be a planetary nebula. ${ }^{1}$ Another which might be an interesting object for spectroscopic observation has the same color index as the planetary nebula but is one magnitude fainter. There appears to be very little space reddening in the direction of this cluster.

I. A. H. Joy, $A p$. $J$. I10, I05, 1949.

Yerkes Observatory, Williams Bay, Wis.

\section{Buscombe, William. Spectrophotometry of early A-type stars.}

Coudé spectra of the main sequence star $\gamma$ Geminorum and of the supergiant $\alpha$ Cygni in the wave-length region $3300-4700 \mathrm{~A}$ have been traced with the new direct-intensity microphotometer at Mount Wilson Observatory. Empirical line-absorption coefficients, derived by other workers from the spectra of stars of both higher and lower excitation temperatures, were used to construct separate curves of growth for the regions on either side of the Balmer continuum. In the ultraviolet, Greenstein's unpublished provisional values of $\log \eta_{0}$ for the hydrogen-poor supergiant $v$ Sagittarii were supple- mented by a few laboratory line strengths for $T i$ II, adjusted to that star's assumed temperature of $15,000^{\circ} \mathrm{K}$. In the visible region, other semi-empirical line strengths from the solar curve of growth and from the metallic-line A star $\tau$ Ursae Majoris were adjusted for abundance and temperature and averaged with the $v$ Sgr material. Analysed with the aid of Wrubel's theoretical curve of growth, the measures of some 400 lines on each of 3 plates for each of the A stars indicate that their excitation temperatures are close to $10,000^{\circ} \mathrm{K}$. Empirical $\log \eta_{0}$ values for the A stars are being derived.

The opacity difference measured across the Balmer continuum corresponds to seeing about I 5 times more atoms above the photosphere in the violet than in the far ultraviolet. This is completely accounted for by continuous $H$ and $H^{-}$absorption at the electron pressures indicated by the ionisation of iron. The ratio of these electron pressures is confirmed by counting the number of Stark-broadened lines in the Balmer series whose cores can be resolved from the confluence of their wings, although the Inglis-Teller formula is more nearly valid for spectrograms of lower dispersion. The very flat transitional portions of the curves of growth indicate a damping constant of at most one-third the classical value.

No significant difference in turbulence is evident between the visible and ultraviolet regions, either from the curves of growth or from the line profiles, whose form is remarkably near Gaussian. The velocities have been corrected for thermal motion, I. $6 \mathrm{~km} / \mathrm{sec}$, and instrumental broadening, half-half-width $4.6 \mathrm{~km} / \mathrm{sec}$. Evidently considerable energy is transferred below the photosphere in eddies with a wave length exceeding the scale height.

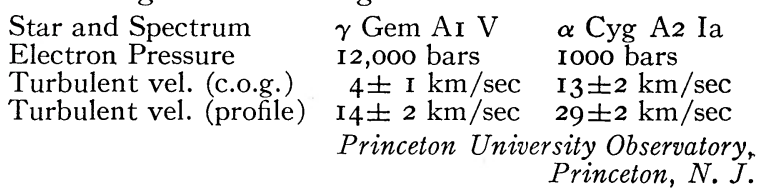

Carpenter, E. F. A directly indicating photoelectric colorimeter.

The a.c. photometer of the Steward Observatory ${ }^{1}$ is converted into a wedge colorimeter in the following simple way:

I. The slotted cylindrical light-chopper surrounding the $\mathrm{IP}_{2} \mathrm{I}$ phototube is replaced by a transparent chopper covered with alternating. and contiguous blue and yellow filters. 